\title{
Laminar Shear Stress Elicit Distinct Endothelial Cell E-Selectin Expression Pattern Via TNF $\alpha$ and IL-1及 Activation
}

\author{
Ryan B. Huang, ${ }^{1}$ Anjelica L. Gonzalez, ${ }^{2}$ Omolola Eniola-Adefeso ${ }^{1}$ \\ ${ }^{l}$ Department of Chemical Engineering, University of Michigan, Ann Arbor, Michigan 48109; \\ telephone: 734-936-0856; fax: 734-764-1761; e-mail: lolaa@umich.edu \\ ${ }^{2}$ Department of Biomedical Engineering, Yale University, New Haven, CT 06520
}

ABSTRACT: The ability to discriminate cell adhesion molecule expression between healthy and inflamed endothelium is critical for therapeutic intervention in many diseases. This study explores the effect of laminar flow on TNF $\alpha$-induced E-selectin surface expression levels in human umbilical vein endothelial cells (HUVECs) relative to IL- $1 \beta$-induced expression via flow chamber assays. HUVECs grown in static culture were either directly (naïve) activated with cytokine in the presence of laminar shear or pre-exposed to $12 \mathrm{~h}$ of laminar shear (shear-conditioned) prior to simultaneous shear and cytokine activation. Naïve cells activated with cytokine in static served as control. Depending on the cell shear history, fluid shear is found to differently affect TNF $\alpha$ induced relative to IL-1 $\beta$-induced HUVEC expression of Eselectin. Specifically, E-selectin surface expression by naïve HUVECs is enhanced in the $8-12 \mathrm{~h}$ activation time range with simultaneous exposure to shear and TNF $\alpha$ (shear$\mathrm{TNF} \alpha$ ) relative to TNF $\alpha$ static control whereas enhanced E-selectin expression is observed in the $4-24 \mathrm{~h}$ range for shear-IL-1 $\beta$ treatment relative to IL- $1 \beta$ static control. While exposure of HUVECs to shear preconditioning mutes shear$\mathrm{TNF} \alpha$-induced E-selectin expression, it enhances or downregulates shear-IL-1 $\beta$-induced expression dependent on the activation period. Under dual-cytokine-shear conditions, IL-1 $\beta$ signaling dominates. Overall, a better understanding of E-selectin expression pattern by human ECs relative to the combined interaction of cytokines, shear profile and history can help elucidate many disease pathologies.

Biotechnol. Bioeng. 2013;110: 999-1003.

(C) 2012 Wiley Periodicals, Inc.

KEYWORDS: inflammation; parallel plate flow chamber; HUVEC; reperfusion injury; vascular-targeting

No conflicts of interest exist for any of the authors.

Ryan B. Huang's present address is Department of Chemical and Biomolecular Engineering, University of Illinois, Urbana, IL 61801.

Correspondence to: 0. Eniola-Adefeso

Contract grant sponsor: American Heart Association

Contract grant number: $0735043 \mathrm{~N}$

Additional supporting information may be found in the online version of this article.

Received 11 June 2012; Revision received 25 September 2012;

Accepted 28 September 2012

Accepted manuscript online 10 October 2012;

Article first published online 18 October 2012 in Wiley Online Library

(http://onlinelibrary.wiley.com/doi/10.1002/bit.24746/abstract)

DOI 10.1002/bit.24746
Endothelial cell (EC) response to various chemical agonists results in the differential surface expression of cell adhesion molecules (CAMs) and is critical to the balance between healthy and pathogenic inflammation response (Aird, 2008). The ability to discriminate between healthy and diseased tissue through these CAM expression patterns can significantly impact the design of vascular-targeted therapy in human diseases. Despite this potential, in vivo EC behavior under chronic inflammation has yet to be fully understood. Though complex in vitro assays have been developed to mimic relevant EC response, these often fail to simulate relevant physiological conditions under which inflammation occurs since the majority of published works is focused on in vitro EC response to either chemical or mechanical stimulus alone. However, most occurrences of inflammation in vivo are characterized by EC exposure to inflammatory cytokines in the simultaneous presence of blood flow-induced shear. The few works that have investigated cytokine stimulation of ECs in the presence of flow (shear-cytokine activation) in vitro have focused on acute time frames likely not relevant for analysis of chronic inflammation (Ji et al., 2008; Sheikh et al., 2003; Tsou et al., 2008). These shear-cytokine studies have also emphasized the expression of intercellular cell adhesion molecule-1 (ICAM-1) rather than the expression of E-selectin, which is arguably the more important molecule in inflammation response due to its significant role in the initial capture of leukocytes to the vascular wall (Ley et al., 2007) and its association with disease progression in many chronic diseases (Roldan et al., 2003). We previously reported that the pattern and magnitude of E-selectin expression on human umbilical vein endothelial cells (HUVECs) induced by recombinant human interleukin-1ß (IL-1 $\beta$ ) are dependent on the shear history of the HUVECs, the magnitude of the simultaneously imposed fluid shear, and the length of exposure to chemical stimulus (Huang and Eniola-Adefeso, 2012). Here, we explore whether previously observed shearIL- $1 \beta$-induced E-selectin surface expression patterns on HUVECs are replicated for long-term tumor necrosis factor 
$\alpha(\mathrm{TNF} \alpha)$ stimulation under similar fluid shear conditions. Concentrations of $10 \mathrm{ng} / \mathrm{mL}$ of TNF $\alpha$ and $0.1 \mathrm{ng} / \mathrm{mL}$ of IL$1 ß$ were used in assays involving these cytokines individually or in combination. These concentrations are 10-fold higher than the TNF $\alpha$ and IL-1ß concentrations that have been previously found to elicit maximum E-selectin expression in static at $4 \mathrm{~h}$ of activation time. E-selectin surface expression by HUVEC activated in static was found to not change at concentrations greater than $0.01 \mathrm{ng} / \mathrm{mL}$ for IL- $1 ß$ (Fig. S1 in the Supplementary Material) and $1 \mathrm{ng} / \mathrm{mL}$ for TNF $\alpha$ (Wyble et al., 1996). Similarly, E-selectin surface expression induced in HUVECs by IL-1ß under shear conditions was also found to not respond to IL-1ß concentration changes in preliminary assays (Fig. S2 in the Supplementary Material). Thus, over-saturating concentrations of cytokines were used to ensure the maximum E-selectin expression in HUVECs.

For naïve HUVECs, as shown in Figure 1, monolayers treated with TNF $\alpha$ under shear conditions $\left(10 \mathrm{dyn} / \mathrm{cm}^{2}\right.$; shear-TNF $\alpha$ ) expressed higher E-selectin density at intermediate time points compared to monolayers treated in static (static-TNF $\alpha$ ). As such, the maximum E-selectin expression occurred in the 8-12 $\mathrm{h}$ range for shear-TNF $\alpha$ activation rather than the typically reported $4-6 \mathrm{~h}$ range for static-TNF $\alpha$ activation. We previously showed via neutrophil adhesion assays that such shear-enhanced Eselectin expression on HUVEC is functional (Huang and Eniola-Adefeso, 2012). The lack of previous reports in the literature of shear enhancing $\mathrm{TNF} \alpha$-signaled $\mathrm{E}$-selectin expression in naïve ECs is likely due to most studies only directly comparing shear-cytokine induced E-selectin expression to static-cytokine activation over a short cytokine activation time (typically $4-6 \mathrm{~h}$ ) where shear-TNF $\alpha$ activation is consistently shown to attenuate E-selectin expression (as was also shown in Fig. 1; Kraiss et al., 2003; Xie et al., 2010). Monolayers subjected to $12 \mathrm{~h}$ of laminar shear at $10 \mathrm{dyn} / \mathrm{cm}^{2}$ (i.e., preconditioning) prior to shear-TNF $\alpha$ activation exhibited significantly lower E-selectin expression at each time point evaluated (up to $12 \mathrm{~h}$ ) relative to the corresponding static-TNF $\alpha$-activated naïve cells. However, when compared to shear-TNF $\alpha$-activated naïve cells (i.e., non-preconditioned), E-selectin expression by preconditioned HUVECs was significantly lower at both the 8 and $12 \mathrm{~h}$ marks but was not significantly different at the $4 \mathrm{~h}$ mark.

The most interesting observation of the present work is in the differential response of preconditioned HUVECs to shear-TNF $\alpha$ compared to shear-IL-1 $\beta$ activation. Although both shear-cytokine treatments elicited the same E-selectin density at $12 \mathrm{~h}$, the E-selectin expression induced on preconditioned cells by shear-TNF $\alpha$ activation was significantly lower than the expression induced by shear-IL-1 $\beta$ at the 4 and $8 \mathrm{~h}$ activation times (Fig. 2). This trend is opposite the trend observed between the two cytokines for static activation of naïve HUVECs where significantly higher E-selectin density was induced with TNF $\alpha$ (Fig. 1) compared to activation with IL-1 $\beta$ (Fig. 2), for all activation times. Moreover, the E-selectin expression

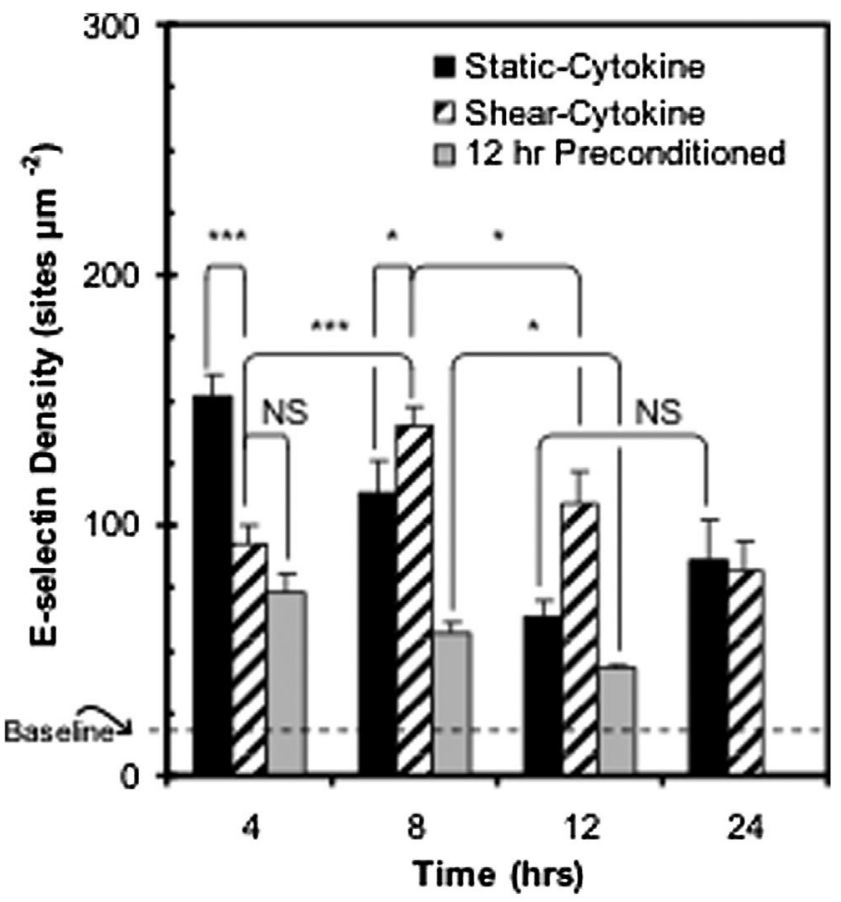

Figure 1. E-selectin site density of TNF $\alpha$ activated monolayers. Naïve HUVEC monolayers were treated with $10 \mathrm{ng} / \mathrm{mL}$ of TNF $\alpha$ for $4,8,12$, or $24 \mathrm{~h}$ either in static (static-cytokine; solid) or in the presence of $10 \mathrm{dyn} / \mathrm{cm}^{2}$ of laminar shear (shearcytokine; diagonal). Some HUVEC monolayers were first preconditioned with fluid shear for $12 \mathrm{~h}$ (at $10 \mathrm{dyn} / \mathrm{cm}^{2}$ ) followed by activation with $10 \mathrm{ng} / \mathrm{mL}$ of TNF $\alpha$ in the presence of $10 \mathrm{dyn} / \mathrm{cm}^{2}$ of laminar shear for up to $12 \mathrm{~h}$ (12 h Preconditioned; gray). The $24 \mathrm{~h}$ shear-cytokine activation of preconditioned monolayer was not evaluated.

induced in preconditioned HUVECs by the $4 \mathrm{~h}$ shear-TNF $\alpha$ activation was 1.3- and 2.1-fold lower than the expression induced in naïve HUVECs by the $4 \mathrm{~h}$ shear-TNF $\alpha$ and staticTNF $\alpha$ activations, respectively (Fig. 1). The opposite was observed for IL- $1 \beta$ activation, where the E-selectin density induced in preconditioned HUVECs by the $4 \mathrm{hr}$ shear-IL-1 $\beta$ activation was 1.5 - and 1.7 -fold higher than the density induced in naïve HUVECs by shear-IL-1 $\beta$ and static-IL-1 $\beta$ activations, respectively (Fig. 2 ). This shear-IL-1 $\beta$ stimulated HUVEC response slightly deviates from a previous report by Sheikh et al. (2005) where HUVECs preconditioned with high laminar shear for $24 \mathrm{~h}$ followed by $4 \mathrm{~h}$ of shear-IL- $1 \beta$ activation were shown to support similar levels of neutrophil adhesion and transmigration relative to static, suggesting similar levels of protein expression. However, the consistently lower E-selectin expression by shear preconditioned HUVECs exposed to shear-TNF $\alpha$ activation relative to activation of naïve HUVECs is in good agreement with similar studies in the literature (Chiu et al., 2004; Cicha et al., 2009; Urschel et al., 2011), and highlights the relevance of our observation that shear preconditioning enhances E-selectin expression by shear-IL-1 $\beta$ activated HUVECs. It is possible that the difference in the shear conditioning time $(12 \mathrm{~h}$ vs. $24 \mathrm{~h})$ is responsible for the discrepancy in 


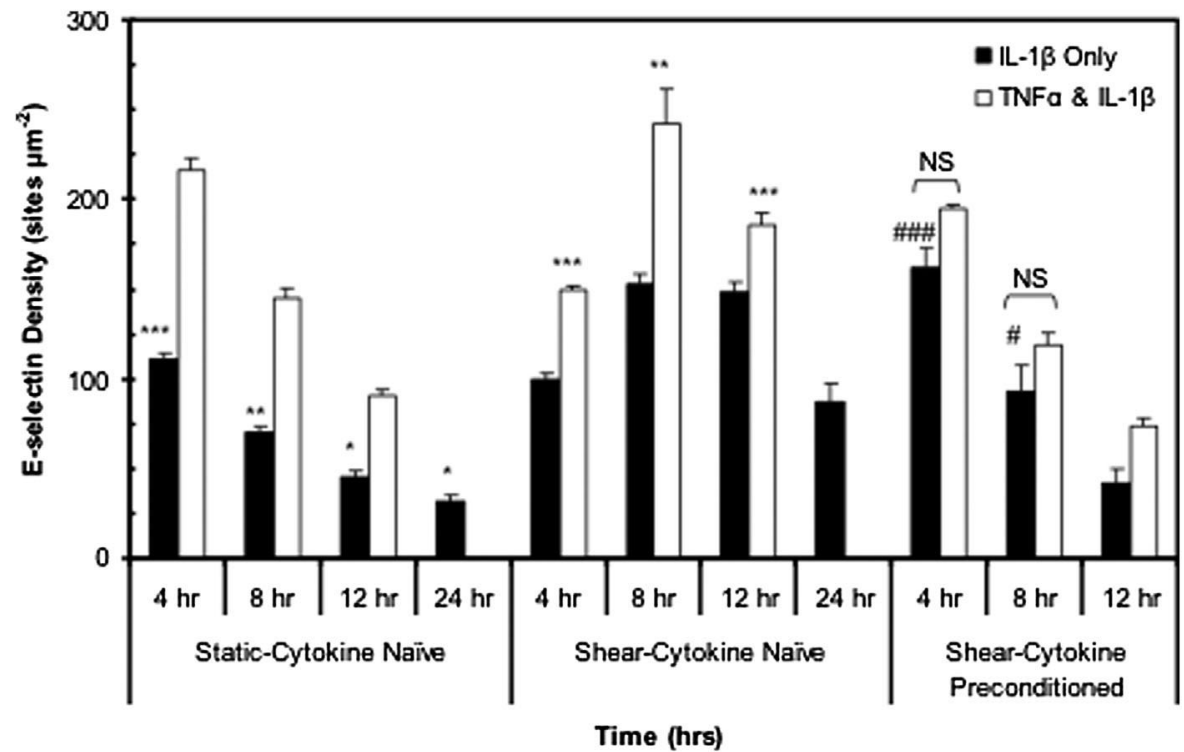

Figure 2. Comparing E-selectin expressions between IL-1 $\beta$ and dual-cytokine activated monolayers. Inflammatory agonists, IL-1 $\beta$ (filled) or both IL-1 $\beta$ and TNF $\alpha$ (clear), were used to activate naïve HUVEC monolayers under static-cytokine (first data set) and $10 \mathrm{dyn} / \mathrm{cm}^{2}$ shear-cytokine (second data set) activation conditions. Some HUVEC monolayers were exposed to shear preconditioned (at $10 \mathrm{dyn} / \mathrm{cm}^{2}$ for $12 \mathrm{~h}$ ) prior to activation via $10 \mathrm{dyn} / \mathrm{cm}^{2}$ shear-cytokine conditions (third data set). The $24 \mathrm{~h}$ static or shear-

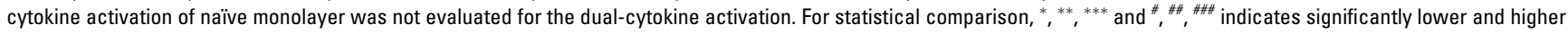
E-selectin expression $(P<0.05, P<0.01, P<0.001)$, respectively, relative to the corresponding TNF $\alpha$ cytokine treatment. All differences between "IL-1 $\beta$ Only" and "IL-1 $\beta$ \& TNF $\alpha$ " are significant, unless otherwise noted via "NS".

the $4 \mathrm{~h}$ shear-IL-1 $\beta$ induced E-selectin expression trend between preconditioned and naïve HUVECs reported here relative to the previous report by Sheikh et al. (2005).

In light of the differential response of preconditioned HUVECs to shear-TNF $\alpha$ and shear-IL- $1 \beta$ stimulation, we sought to determine which of the two cytokines dominate, if at all, when cells are subjected to both agonists under shear conditions particularly since IL- $1 \beta$ has been previously shown to inhibit the TNF $\alpha$ stimulatory effect on ECs in static (Holtmann and Wallach, 1987). Thus, naïve and shear preconditioned HUVECs were co-activated with both IL- $1 \beta$ and TNF $\alpha$ at the same concentrations as used for individual stimulations. For static activated naïve HUVECs, maximum E-selectin density was observed at $4 \mathrm{~h}$ with dual-cytokine activation (Fig. 2) similar to observations in cells activated in static by either IL- $1 \beta$ or TNF $\alpha$. At all tested time points, E-selectin levels for staticdual cytokine activated naïve HUVECs were only slightly less $(\sim 18 \pm 1.3 \%$ lower $)$ than the additive sum of the Eselectin densities observed for individual cytokine-activated cells. When naïve HUVECs were simultaneously activated under shear and dual-cytokine, maximum expression occurred between 8 and $12 \mathrm{~h}$ similar to observations with single cytokine activations. Again, the E-selectin expression levels for shear-dual cytokine-activated naïve HUVECs are less $(\sim 22 \pm 5.7 \%)$ than the additive sum of the levels observed for single cytokine-activated cells at all time points.
For shear preconditioned monolayers (Fig. 2), sheardual-cytokine activation yielded maximum E-selectin expression at $4 \mathrm{~h}$. Expression then progressively decreased at longer activation times. Furthermore, the E-selectin expressions induced in preconditioned HUVECs by sheardual cytokine activation were not significantly different from the levels induced by shear-IL- $1 \beta$ stimulation at the 4 and $8 \mathrm{~h}$ time points while being on average 2.1 -fold higher than expression by shear-TNF $\alpha$. This observation would suggest an attenuated HUVEC response to TNF $\alpha$ stimulation in the presence of IL-1 $\beta$. Indeed, a few published reports have shown that IL- $1 \beta$ and shear each independently negatively regulate EC response to TNF $\alpha$ stimulation. For example, Holtmann and Wallach (1987) reported that exposure of ECs to IL-1 $\beta$ in static conditions downregulates TNF $\alpha$ receptors, while Yamawaki et al. (2003) showed that pre-exposure of ECs to long-term high laminar shear inhibited TNF $\alpha$ activation of the JNK and p38 MAPK pathways preventing TNF receptors (TNFR) from associating with TNFR-associated factor-2. Thus, it follows that HUVEC E-selectin expression under shear-dual cytokine stimulation is likely dominated by IL- $1 \beta$. In other words, the effect of long-term HUVEC exposure to laminar shear synergistically combines with the effect of IL- $1 \beta$ to push Eselectin expression for dual-cytokine stimulations towards values observed for IL-1 $\beta$ stimulation alone.

To more clearly discern the effects of shear separate from cytokine stimulation, we normalized the E-selectin expression levels obtained for shear-cytokine stimulations to the 
complementary data points under static conditions (normalized data is obtained from assays that directly observed differences between shear-cytokine activation and corresponding static activation controls). Figure 3 reveals that the effects of shear being present during cytokine activation for naïve HUVEC monolayers are quite distinct from the effects for preconditioned HUVECs. Relative to static, naïve HUVECs exposed to $4 \mathrm{~h}$ of shear-TNF $\alpha$ or shear-dual cytokine stimulation were less responsive to activation, eliciting a 1.7- and 1.4-fold reduction $(P<0.001$ for both) in E-selectin density, respectively, whereas shear-IL-1 $\beta$ activation resulted in virtually no reduction. Over the $8-12 \mathrm{~h}$ range, cytokine stimulation under shear generally enhanced E-selectin expression by naïve monolayers relative to static conditions. All shear-cytokine stimulation of naïve HUVECs resulted in increased expression of E-selectin relative to previous time points, with IL-1 $\beta$-treated monolayers being the most responsive over time. Conversely, the response to shear-cytokine stimulation by preconditioned cells differs depending on the type of cytokine treatment. Shear preconditioning induced a clear protection against inflammation (an average of 1.55 -fold reduction in E-selectin density, $P=0.019$ ) via shear-TNF $\alpha$ stimulation over the $12 \mathrm{~h}$ activation period relative to static-TNF $\alpha$ stimulation of naïve cells. However, preconditioning promoted a timedependent positive-to-neutral response to shear-IL-1 $\beta$

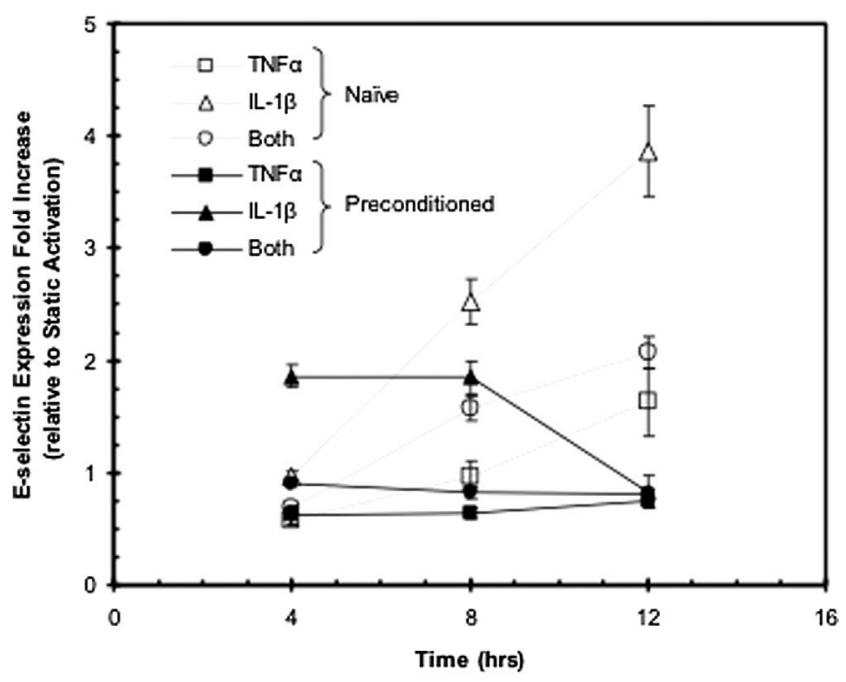

Figure 3. Isolated effects of shear on endothelial cells under cytokine stimulation. E-selectin densities for naïve (dotted lines, clear) and $12 \mathrm{~h}$ preconditioned (solid lines, filled) monolayers stimulated via simultaneous exposure to $10 \mathrm{dyn} / \mathrm{cm}^{2}$ of laminar shear and TNF $\alpha$ ( $\square$, squares), IL-1 $\beta$ ( $\Delta$, triangles), or dual-cytokine $(\bigcirc$, circles) were normalized to fold increases over corresponding E-selectin expression of static activated naïve cells. Note: Normalized data sets are derived using some of the experimental data included in Figures 1 and 2. Specifically, the normalized data includes only E-selectin expression from assays that directly compared shear-cytokine activation with their corresponding static activation controls (monolayer of HUVEC were plated from the same T-75 flask and experiment done on the same day) whereas the data presented in Figures 1 and 2 are an average of all experimental data regardless of whether they were done head-to-head with a static experiment. stimulation relative to static with a 1.8 fold increase in Eselectin expression at 4 and $8 \mathrm{~h}$ marks and no change at $12 \mathrm{~h}$ ( $P=0.39$; relative to static expression in naïve cells). For dual cytokine stimulation, preconditioning only induced a minimal, diminished response $(\sim 1.17$-fold reduction in Eselectin density; $P>0.05$ at $12 \mathrm{~h}$ ) for activation under shear conditions relative to static activation of naïve cells, especially at later time points.

Overall, while the observed E-selectin expression pattern in naïve HUVECs is likely not relevant in healthy vessels, these EC phenotypes may exist in some disease models. This may be the case for ischemia/reperfusion injury characterized by significant infiltration of inflammatory cells and tissue damage specifically associated with the restoration of flow following an ischemic disruption. In this disease, ECs likely undergo a physical experience mimicked here by the shear-cytokine experimental condition, that is, ECs in vivo acquire a naive phenotype in the prolonged absence of flow and hence exhibit an enhanced pro-inflammatory phenotype upon restoration of flow. In this case, the presence of cytokines in combination with disturbed flow (i.e., "stop and go" flow pattern) could enhance inflammatory response leading to tissue damage. The fact that the presence of IL-1 $\beta$ alone or in dual cytokine stimulation with TNF $\alpha$ is observed to be more pro-inflammatory than TNF $\alpha$ alone is consistent with the previous indication that this cytokine has a major role in reperfusion injury (Boutin et al., 2001).

In conclusion, the presented work suggests that shear history coupled with the type and number of cytokines present dictates temporal E-selectin expression level in HUVECs. While shear preconditioning consistently induces a protective mechanism against $\mathrm{TNF} \alpha$ activation, this protection is eliminated under dual-cytokine stimulation or reversed with IL-1 $\beta$ activation alone. This would suggest that IL-1 $\beta$ signaling of inflammatory response is significantly more robust than that of TNF $\alpha$ under the most quiescent physical circumstances. Overall, a better understanding of the synergy between shear and different cytokine in inflammation response is critical for better understanding of many disease pathologies, which could pave way for novel treatments.

\section{Materials and Methods}

Endothelial cells were isolated from fresh umbilical cords as previously described (Huang and Eniola-Adefeso, 2012). For all assays, HUVECs were subcultured at confluent density onto gelatin-coated glass coverslips (Warner Instruments, Hamden $\mathrm{CT}$ ) and incubated with standard culture media at $37^{\circ} \mathrm{C}$ for at least $36 \mathrm{~h}$ in a $5 \% \mathrm{CO}_{2}$ humidified incubator to allow for the development of tight junctions prior to use. Only zero to third generation passages were used. All human tissue protocols were approved by the University of Michigan Internal Review Board and in line with the standards set by the Helsinki Declaration. 
Two distinct types of HUVEC monolayers were used in assays: "naïve" monolayers represent ones that were grown in culture under static conditions and used directly in assays upon confluence and "pre-conditioned" monolayers represent ones that were grown to confluence under static conditions but were exposed to $12 \mathrm{~h}$ of laminar shear only at 10 dyne $/ \mathrm{cm}^{2}$ prior to use in assays. For static assays, naïve HUVEC monolayers on glass coverslips were exposed to a single dose of cytokine in the absence of flow for the desired length of time up to $24 \mathrm{~h}$. For flow assay, HUVEC monolayers, naïve or preconditioned, on glass coverslips were attached to straight channel parallel plate flow chambers (GlycoTech, Gaithersburg, MD) and were exposed to a fixed wall shear stress of $10 \mathrm{dyn} / \mathrm{cm}^{2}$ via flow of cell culture media in the presence of cytokine for the desired time - up to 12 or $24 \mathrm{~h}$ for preconditioned or naïve monolayers, respectively. The same cytokine concentrations used in static assays were used for flow assays. Wall shear stress $\left(\mathrm{dyn} / \mathrm{cm}^{2}\right)$ in the flow chamber was computed as previously described (Charoenphol et al., 2010; EniolaAdefeso et al., 2009). E-selectin surface expression on HUVEC was quantified via immunofluorescence (Huang and Eniola-Adefeso, 2012). All experiments were performed in at least triplicate; averages were calculated and standard errors are shown unless otherwise noted. Significance was determined using Student's $t$-test for one-on-one comparisons and one-way ANOVA and Bonferroni post-test (GraphPad Prism, San Diego, CA) for comparison between groups. Data were considered significant for $P$ values $<0.05$ (i.e., 95\% confidence interval).

This work was supported by a grant from the American Heart Association $(0735043 \mathrm{~N})$ to O.E.A.

\section{References}

Aird WC. 2008. Endothelium in health and disease. Pharmacol Rep 60(1):139-143.

Boutin H, LeFeuvre RA, Horai R, Asano M, Iwakura Y, Rothwell NJ. 2001. Role of IL-1alpha and IL-1beta in ischemic brain damage. J Neurosci 21(15):5528-5534.

Charoenphol P, Huang RB, Eniola-Adefeso O. 2010. Potential role of size and hemodynamics in the efficacy of vascular-targeted spherical drug carriers. Biomaterials 31(6):1392-1402.

Chiu JJ, Lee PL, Chen CN, Lee CI, Chang SF, Chen LJ, Lien SC, Ko YC, Usami S, Chien S. 2004. Shear stress increases ICAM-1 and decreases VCAM-1 and E-selectin expressions induced by tumor necrosis factor[alpha] in endothelial cells. Arterioscler Thromb Vasc Biol 24(1):73-79.
Cicha I, Beronov K, Ramirez EL, Osterode K, Goppelt-Struebe M, Raaz D, Yilmaz A, Daniel WG, Garlichs CD. 2009. Shear stress preconditioning modulates endothelial susceptibility to circulating TNF-alpha and monocytic cell recruitment in a simplified model of arterial bifurcations. Atherosclerosis 207(1):93-102.

Eniola-Adefeso O, Huang RB, Smith CW. 2009. Kinetics of LFA-1 mediated adhesion of human neutrophils to ICAM-1-Role of E-selectin signaling post-activation. Ann Biomed Eng 37(4):737-748.

Holtmann H, Wallach D. 1987. Down regulation of the receptors for tumor necrosis factor by interleukin 1 and 4 beta-phorbol-12-myristate-13acetate. J Immunol 139(4):1161-1167.

Huang RB, Eniola-Adefeso O. 2012. Shear stress modulation of IL-1betainduced E-selectin expression in human endothelial cells. PLoS ONE 7(2):e31874.

Ji JY, Jing H, Diamond SL. 2008. Hemodynamic regulation of inflammation at the endothelial-neutrophil interface. Ann Biomed Eng 36(4):586595.

Kraiss LW, Alto NM, Dixon DA, McIntyre TM, Weyrich AS, Zimmerman GA. 2003. Fluid flow regulates E-selectin protein levels in human endothelial cells by inhibiting translation. J Vasc Surg 37(1):161168.

Ley K, Laudanna C, Cybulsky MI, Nourshargh S. 2007. Getting to the site of inflammation: The leukocyte adhesion cascade updated. Nat Rev Immunol 7(9):678-689.

Roldan V, Marin F, Lip GY, Blann AD. 2003. Soluble E-selectin in cardiovascular disease and its risk factors. A review of the literature. Thromb Haemost 90(6):1007-1020.

Sheikh S, Rainger GE, Gale Z, Rahman M, Nash GB. 2003. Exposure to fluid shear stress modulates the ability of endothelial cells to recruit neutrophils in response to tumor necrosis factor-alpha: A basis for local variations in vascular sensitivity to inflammation. Blood 102(8):28282834.

Sheikh S, Rahman M, Gale Z, Luu NT, Stone PC, Matharu NM, Rainger GE, Nash GB. 2005. Differing mechanisms of leukocyte recruitment and sensitivity to conditioning by shear stress for endothelial cells treated with tumour necrosis factor-alpha or interleukin-1beta. Br J Pharmacol 145(8):1052-1061.

Tsou JK, Gower RM, Ting HJ, Schaff UY, Insana MF, Passerini AG, Simon SI. 2008. Spatial regulation of inflammation by human aortic endothelial cells in a linear gradient of shear stress. Microcirculation 15(4):311323.

Urschel K, Garlichs CD, Daniel WG, Cicha I. 2011. VEGFR2 signalling contributes to increased endothelial susceptibility to TNF-alpha under chronic non-uniform shear stress. Atherosclerosis 219(2):499-509.

Wyble CW, Desai TR, Clark ET, Hynes KL, Gewertz BL. 1996. Physiologic concentrations of TNFalpha and IL-1beta released from reperfused human intestine upregulate E-selectin and ICAM-1. J Surg Res 63(1):333-338.

Xie LX, Durairajan SS, Lu JH, Liu CL, Kum WF, Wang Y, Koo I, Wu WK, Han D, Lao F, et al. 2010. The effect of salvianolic acid B combined with laminar shear stress on TNF-alpha-stimulated adhesion molecule expression in human aortic endothelial cells. Clin Hemorheol Microcirc 44(4):245-258.

Yamawaki H, Lehoux S, Berk BC. 2003. Chronic physiological shear stress inhibits tumor necrosis factor-induced proinflammatory responses in rabbit aorta perfused ex vivo. Circulation 108(13):1619-1625. 